Abstract P25 Table 1 Patients recommended treatment vary according to guideline

\begin{tabular}{|c|c|c|c|c|}
\hline & $\begin{array}{l}\text { Pre-BMD } \\
\text { FRAX }{ }^{\circledR} \text { (NOGG) }\end{array}$ & $\begin{array}{l}\text { Post- BMD } \\
\text { FRAX }{ }^{\circledR} \text { (NOGG) }\end{array}$ & BSG & NICE \\
\hline Number of relevant patients & 103 & 103 & 132 & 43 \\
\hline Patients requiring DXA & 32 & NA & 132 & NA \\
\hline \multicolumn{5}{|c|}{ Anti-osteoporotic treatment recommended } \\
\hline Total & 9 & 16 & 25 & 3 \\
\hline High risk (FRAX® $+B M D)$ & 8 & 16 & 9 & 1 \\
\hline Low risk (FRAX® + BMD) & 1 & $\mathrm{~N} / \mathrm{A}$ & 16 & 2 \\
\hline \multicolumn{5}{|l|}{ Treatment not recommended } \\
\hline Total & 94 & 87 & 107 & 40 \\
\hline High risk $\left(F R A X\left({ }^{\circledR}+B M D\right)\right.$ & 8 & $\mathrm{~N} / \mathrm{A}$ & 11 & 4 \\
\hline Low risk (FRAX® + BMD) & 86 & 87 & 96 & 36 \\
\hline
\end{tabular}

categorises patients to low, intermediate or high risk according to set thresholds by the National Osteoporosis Guidelines Group (NOGG) and recommend lifestyle-advice, DXA or anti-osteoporosis treatment respectively (intermediate patients are rescored post-BMD to either high or low risk). Cirrhosis contributes to calculated fracture risk.

Aim To determine what proportion of our patients would be eligible for DXA and recommended treatment under BSG, NICE and $\mathrm{FRAX}^{\circledR} /$ NOGG guidelines. To calculate the correlation of the pre-BMD FRAX ${ }^{\circledR}$ scores with the post-BMD FRAX ${ }^{\circledR}$ scores.

Method We interrogated our outpatient database of cirrhotic patients and calculated the FRAX ${ }^{\circledR}$ score pre and post-BMD measurement. For each guideline we calculated the number of: patients eligible for treatment; high risk patients left untreated and low risk patients unnecessarily treated.

Results Of 132 patients $(58 \%$ male) with cirrhosis (mixed aetiology), 43 had normal $\mathrm{BMD}, 64$ were osteopaenic and 25 osteoporotic. 103/132 patients (43 postmenopausal women and 60 men $>50$ years) were eligible for FRAX ${ }^{\circledR}$. Of these, 64/103 were estimated low, 33/103 intermediate, and 6/103 at high risk of fracture. Of those at low risk (and therefore not required to undergo DXA scanning (NOGG)), 8 were reclassified as high risk post-BMD (one patient was reclassified from high to low risk). Patients eligible for treatment: pre-BMD FRAX ${ }^{\circledR} /$ NOGG 10/103, post-BMD FRAX ${ }^{\circledR}$ / NOGG 17/103, BSG 25/132, NICE 3/45. Low risk patients unnecessarily treated: pre-BMD FRAX ${ }^{\circledR} /$ NOGG 1 , BSG 16, NICE 2. High risk patients not recommended to receive treatment: 8,11 , and 4 . Pre-BMD FRAX ${ }^{\circledR}$ correlates well with post-BMD FRAX: Spearman's coefficient $r=0.76$, sensitivity of $47.5 \%$, specificity $98.8 \%$, PPV $90 \%$ and NPV $89 \%$ for correctly risk stratifying patients.

Conclusion BSG guidance does not incorporate clinical risks and is likely to expose some patients to unnecessary tests, or treatment. NICE is not widely applicable and does not suggest when to DXA. Cirrhosis is not listed as a risk factor, thus underestimating fracture risk. FRAX ${ }^{\circledR} /$ NOGG accurately identifies high risk patients and which group to DXA. Scanning only intermediate risk patients saves unnecessary scans, but in our cohort fails to identify 8/94 (8.5\%)

Abstract P25 Table 2 Comparison of pre-BMD and postBMD FRAX

\begin{tabular}{lrlc}
\hline & & \multicolumn{2}{l}{ Post-BMD FRAX } \\
\cline { 3 - 4 } Pre-BMD FRAX $^{\circledR}$ & & Low risk & High risk \\
\hline Low risk & 65 & 57 & 8 \\
Intermediate risk & 32 & 29 & 3 \\
High risk & 6 & 1 & 5 \\
Total & 103 & 87 & 16 \\
\hline
\end{tabular}

Abstract P25 Table 3 Prevalence of clinical risk factors

\begin{tabular}{lll}
\hline Clinical risk factors & $\begin{array}{l}\text { Males } \\
\mathbf{n = 7 6}(\%)\end{array}$ & $\begin{array}{l}\text { Female } \\
\mathbf{n = 5 6}(\%)\end{array}$ \\
\hline 0 & 0 & 0 \\
$\geq 1$ & $31(41)$ & $23(41)$ \\
$\geq 2$ & $45(59)$ & $33(59)$ \\
$\geq 3$ & $10(13)$ & $8(14)$ \\
$\geq 4$ & $1(1.3)$ & 0 \\
\hline
\end{tabular}

high risk patients. Male smokers with alcoholic cirrhosis were overrepresented in this group. Similarly to other cohorts, Pre-BMD FRAX $^{\circledR}$ has a low sensitivity but is highly specific when compared to post-FRAX ${ }^{\circledR}$ BMD.

\section{P26 RAPID IDENTIFICATION, TRIAGE AND MANAGEMENT OF ALCOHOL RELATED HOSPITAL ADMISSIONS}

doi:10.1136/gutjnl-2011-300857a.26

${ }^{1} \mathrm{R}$ J Aspinall, ${ }^{2} \mathrm{~S}$ Atkins, ${ }^{2} \mathrm{~K}$ Rackham, ${ }^{2} \mathrm{~T}$ Barratt, ${ }^{3} \mathrm{P}$ Schmidt, ${ }^{4} \mathrm{~J}$ Ward, ${ }^{2}$ Donna Bowman. ${ }^{1}$ Department of Gastroenterology and Hepatology, Queen Alexandra Hospital, Portsmouth, UK; ${ }^{2}$ Alcohol Liaison Service, Queen Alexandra Hospital, Portsmouth, UK; ${ }^{3}$ Medical Admissions Unit, Queen Alexandra Hospital, Portsmouth, UK; ${ }^{4}$ WMC Limited

Introduction Portsmouth Hospitals NHS Trust has the highest rate of alcohol related hospital admissions in the South East of England (1993 per 100000 population for 2009/2010). Admissions with complications of cirrhosis have doubled in under 4 years and recorded deaths from liver cirrhosis exceed the regional and national averages. As part of a comprehensive strategy to tackle the rising burden of alcoholic cirrhosis, a dedicated team of alcohol nurse specialists has been deployed to identify all alcohol related admissions at the earliest time point and initiate management. We conducted a prospective evaluation of the patients referred to the service over a 4-month period.

Aim To determine the demographic pattern of patients admitted with alcohol related illnesses in a single large hospital serving a population of 650000 .

Method All patients at the Queen Alexandra Hospital with an alcohol related admission identified during the study period were referred to the specialist nursing service and recorded on a secure database. A comprehensive clinical assessment was made by a team of 3 specialist nurses working to agreed protocols. The WHO Alcohol Use Disorders Identification Test (AUDIT) score was recorded along with data regarding age of first alcohol exposure, regular usage and problem drinking. Liver related morbidity was determined by clinical assessment, standard blood parameters and by calculation of Model for Endstage Liver Disease (MELD) and Glasgow Alcoholic Hepatitis (GAHS) scores.

Results During the study period, 495 new patients were referred to the service. $71 \%$ were identified and referred in the ED or MAU, the remainder from inpatient wards. Mean age was 50 years (range 18-91) and $72 \%$ were male. $97.5 \%$ of patients were of white British ethnicity and only $11 \%$ were in regular employment. There were no gender differences in the proportion of younger drinkers $<40$ years $(20 \%$ in both sexes). Overall, $12 \%$ of patients referred with alcohol misuse disorders were aged over 70 years. Our cohort demonstrated heavy current alcohol misuse with a mean weekly consumption of 229 units at admission (range 8-860) and $50 \%$ of patients recorded the maximum AUDIT score of 40 . The mean age of first drinking alcohol was 11 years (range 3-26) and the mean age of establishing regular use was 22 (range 11-65). Laboratory 
parameters identified a high proportion of patients with subclinical evidence of liver injury. Supervised detoxification was completed in 170 patients.

Conclusion Alcohol related hospital admissions can be rapidly identified and managed by a dedicated specialist nursing team working in conjunction with hepatologists and acute physicians. Our cohort demonstrated strong links between poor socioeconomic status and early age of exposure to alcohol, age of regular alcohol misuse and long-term excessive drinking. We have now instituted formal screening of all hospital admissions in ED and MAU with direct electronic referral to the liaison service where alcohol misuse is a factor.

\section{P27 REDUCTION IN ALANINE AMINOTRANSFERASE VALUES IN DIABETIC PATIENTS RECEIVING LONG-TERM ORAL ANTIBIOTICS: DOES MODULATION OF GUT FLORA AFFECT HEPATIC INFLAMMATION IN NON-ALCOHOLIC FATTY LIVER DISEASE?}

doi:10.1136/gutjnl-2011-300857a.27

'L Leach, ' J F L Cobbold, ' J Valabhji, ${ }^{1} \mathrm{M}$ R Thursz. ${ }^{1}$ Hepatology and Gastroenterology section, Department of Medicine, St Mary's Hospital, Imperial College London; ${ }^{2}$ Department of Diabetes, St Mary's Hospital, Imperial College Healthcare NHS Trust

Introduction The gut microbiota may play a role in host metabolic processes and have been linked to the pathophysiology of nonalcoholic fatty liver disease (NAFLD). Murine studies have suggested beneficial effects of modulating the gut microbiota using antibiotics, however studies have not looked at the effect of long-term antibiotic therapy in humans.

Aim To investigate the effects of prolonged broad-spectrum antibiotics in patients attending the diabetic foot clinic who are likely to have NAFLD. It was hypothesised that long-term oral antibiotic administration would result in a decrease in serum alanine aminotransferase (ALT) values after 3 months compared to baseline. Method 43 diabetic patients without known hepatic comorbidity, who had taken a minimum of 3 months' broad-spectrum oral antibiotics (predominantly co-amoxiclav) in the diabetic foot clinic were identified retrospectively. Demographic and clinical data were extracted from clinic letters, patient notes, and computer databases. The primary outcome measure was the change in ALT value at day 90 of antibiotics compared to day 0 . Other outcome measures were a change in patient weight, glycosylated haemoglobin (HbA1c), white cell count, bilirubin and alkaline phosphatase at day 90 from day 0 . The wilcoxon signed rank test was used for paired non-parametric data.

Results All patients with abnormal ALT at baseline $(n=6)$ had a significant decrease in ALT from median $43(41-48) \mathrm{U} / \mathrm{l}$ on day 0 , to $32(29-35) \mathrm{U} / 1$ on day $90(\mathrm{p}=0.03) .37$ patients who had a normal ALT at baseline had a small but significant increase in ALT from median $17(13-23) \mathrm{U} / 1$ on day 0 , to $20(14-28) \mathrm{U} / \mathrm{l}$ on day 90 $(p=0.02)$. In the 20 patients with paired HbA1c values, there was a statistically significant decrease in $\mathrm{HbA1c}$ with antibiotic use $(p=0.03)$. There were no significant associations in change in ALT at 90 days with weight change, white cell count, bilirubin, alkaline phosphatase, antibiotic regime or other medications.

Conclusion The robust decrease in ALT values in patients with abnormal baseline ALTs (and therefore likely to have NAFLD) provides indirect evidence that modulation of gut microbiota with oral antibiotics may reduce hepatic inflammation in NAFLD. The possibility of sepsis-induced liver damage accounting for the changes is countered by no change in white cell count, bilirubin or alkaline phosphatase, while reduction in HbA1c without weight change points to reduced insulin resistance. Prospective studies on patients with biopsy-proven non-alcoholic steatohepatitis are awaited.

\section{P28 DEFECTIVE T-REGULATORY FUNCTION IN AUTOIMMUNE HEPATITIS MAY PARTIALLY DERIVE FROM A PRO- INFLAMMATORY SKEWING OF GAL9+T-REGS}

doi:10.1136/gutjnl-2011-300857a.28

R Liberal, G Mieli-Vergani, D Vergani, M S Longhi. King's College London School of Medicine

Introduction In autoimmune hepatitis (AIH) $\mathrm{CD} 4{ }^{+} \mathrm{CD} 25^{+}$regulatory T-cells (T-regs) are defective in their ability to control CD4 T-cell effector function. T-regs express Galectin9 (Ga19), a $\beta$-galactosidase-binding-protein that inhibits Th1-mediated immuneresponses by binding the T-cell-immunoglobulin-and-mucindomain3 (Tim-3) on CD4 effector cells. In AIH T-regs express reduced levels of Gal9.

Aim To characterise lineage-specific transcription factor and cytokine profiles of peripheral-blood-derived $\mathrm{Gal}^{+} \mathrm{T}$-regs in $\mathrm{AIH}$.

Method 34 ANA/SMA+ patients $(24$ females; median age: 14.6 years) and 17 healthy subjects (HS, 12 females, median age: 29 years) were studied. The phenotype of circulating T-regs was determined by cytofluorimetry using CD4, CD25 and CD127 monoclonal antibodies. The frequency of cells positive for Gal9, FOXP3, T-bet, GATA3 and RORC and that of cells producing IFN $\gamma$, IL-10, TGF- $\beta$ and IL-17 were determined by intracellular staining. Treg suppressor function was evaluated in a proliferation assay following co-culture with CD25 ${ }^{-}$Tim $-3^{+}$and CD25 ${ }^{-}$Tim- $-3^{-}$ autologous target cells.

Results Within $\mathrm{Gal}^{+}$cells the frequency of: (1) $\mathrm{FOXP3}^{+}$cells was lower in AIH than in HS (14.4 \pm 2 vs 42.8 $\pm 3.1, \mathrm{p}<0.001)$; (2) T-bet ${ }^{+}$, GATA3 $^{+}$and RORC ${ }^{+}$cells was similar in AIH and HS; (3) IL-10producing cells was lower in AIH than in HS $(5.1 \pm 0.6$ vs $9.1 \pm 0.5$, $\mathrm{p}<0.001$ ) but higher than in the Ga19- ${ }^{-}$-reg fraction for both (AIH: $5.1 \pm 0.6$ vs $2.3 \pm 0.2, p=0.001$; HS: $9.1 \pm 0.5$ vs $3.3 \pm 0.4, p<0.001)$; (4) TGF- $\beta$-producing cells was lower in AIH than in HS $(6.4 \pm 0.7$ vs $8.1 \pm 0.4, p=0.04)$; (5) IFN $\gamma$ - and IL-17-producing cells was higher in AIH than in HS (IFN $\gamma: 4.4 \pm 0.6$ vs $2.1 \pm 0.3, p=0.002$; IL-17: $4.1 \pm 0.6$ vs $1.8 \pm 0.2, \mathrm{p}=0.002)$. Treatment with anti-IL-10 neutralising antibodies reduced T-reg ability to suppress $\mathrm{CD} 25^{-} \mathrm{Tim}-3^{+}$cell proliferation (AIH: $42 \%$ inhibition in the absence of antibodies vs $36 \%$ in their presence, $p=0.06$; HS: $56 \%$ vs $48 \%, p=0.04$ ), while did not affect $\mathrm{CD} 25^{-}$Tim $-3^{-}$cell proliferation.

Conclusion A skewing towards a pro-inflammatory phenotype and a reduced proportion of $\mathrm{FOXP3}^{+}$and IL-10-producing cells within $\mathrm{Gal9}^{+}$T-regs may contribute to defective immunoregulation in $\mathrm{AIH}$ The reduction of $\mathrm{Gal9}^{+} \mathrm{T}$-reg suppression following anti-IL-10 blockade both in health and AIH, suggests a role for IL-10 in Gal9 ${ }^{+} \mathrm{T}$-cell immune-regulatory function.

\section{P29 REDUCED EXPRESSION OF TIM-3 RENDERS TH1 AND TH17 EFFECTOR CELLS LESS AMENABLE TO T-REG CONTROL IN AUTOIMIMUNE HEPATITIS}

doi:10.1136/gutjnl-2011-300857a.29

R Liberal, G Mieli-Vergani, D Vergani, M S Longhi. King's College London School of Medicine

Introduction In autoimmune hepatitis (AIH), CD4 effector immune responses are permitted by defective $\mathrm{CD} 4{ }^{+} \mathrm{CD} 25^{+}$regulatory $\mathrm{T}$-cells (T-regs). In murine studies apoptosis of Th1 effector cells is mediated by binding of $\mathrm{T}$-cell-immunoglobulin-and-mucin-domain3 (Tim-3) on their surface to Galectin-9 (Ga19) expressed by T-regs. In $\mathrm{AIH}, \mathrm{Tim}-3$ is down-modulated on CD4 effector cells.

Aim To test the frequency of Tim $-3^{+}$cells within the Th1, Th2 and Th17 subsets and to evaluate whether Tim-3 expression by CD4 effectors affects their responsiveness to T-reg control. 